\title{
Prolonged survival in a patient with choroidal metastases from urothelial bladder cancer
}

\author{
Kirsty L.Wiltshire, MBBS, FRANZCR; ${ }^{*}$ Norman Laperriere, MD, FRCPC; ${ }^{\dagger}$ \\ Robert G. Bristow, MD, PhD, FRCPC ${ }^{\dagger}$
}

\begin{abstract}
Choroidal metastases secondary to urothelial carcinoma are extremely rare and are usually associated with an extremely poor prognosis. We present a case of an 88-year-old man with newly diagnosed urothelial carcinoma of the bladder who presented with acute loss of vision before commencing definitive concurrent chemoradiotherapy to the bladder. Ophthalmological examination demonstrated bilateral choroidal metastases. He received palliative radiotherapy to the orbits and completed his planned radiotherapy to the bladder. He remained disease-free at last follow-up 4 years after the completion of treatment. We review the literature particularly with regard to diagnosis and management of choroidal metastases. Choroidal metastases should be considered in a patient with a history of urothelial cancer presenting with new onset of eye symptoms.
\end{abstract}

Can Urol Assoc J 2009;3(4):E36-E38.

\section{Introduction}

Choroidal metastases are the most common intraocular

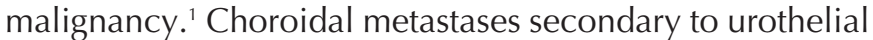
cancers, however, are extremely rare and are usually associated with an extremely poor prognosis. We report an interesting case of a man with choroidal metastases secondary to urothelial cancer of the bladder.

\section{Case report}

An 88-year-old man was referred for management of bladder cancer. He presented with a short history of hematuria and right flank pain. Computed tomography of the abdomen revealed mild pelvicalyceal dilatation and mild ureteric dilatation on the right side down to the level of the bladder. No obvious cause was evident. He underwent a cystoscopy and was found to have a large sessile bladder tumour about 2 to $3 \mathrm{~cm}$ in size visualized in the midline and slightly to the right of the trigone and bladder base. This was resected and pathology demonstrated a transitional cell carcinoma, grade 3 , invading the muscular layer of the bladder wall.
Bone scan demonstrated no evidence of distant metastatic disease.

The patient was seen at Princess Margaret Hospital (PMH) and further staging investigations were undertaken. Radiography of the chest showed no evidence of pulmonary metastases. Magnetic resonance imaging (MRI) of the pelvis performed about 7 weeks after resection of the bladder tumour demonstrated a posterior bladder wall mass involving the right ureteric orifice with no definite evidence of extravesical extension. Also noted were multiple polypoid nodules anteriorly and inferiorly in the bladder lumen. There were multiple small right pelvic lymph nodes and 2 external iliac lymph nodes were mildly enlarged at 1.0 and $1.1 \mathrm{~cm}$. The patient was thus staged as T3a N1 M0 transitional cell carcinoma of the bladder.

The patient's medical history was significant for hypertension and chronic obstructive pulmonary disease. Risk factors for bladder cancer included working in the leather industry and cigarette smoking with a 50 pack-year history. He had a history of bilateral cataract extractions with posterior capsulotomies and decreased vision in the right eye secondary to age-related macular degeneration.

The patient was scheduled for radical external beam radiotherapy (EBRT) with concurrent cisplatin chemotherapy. About 1 week before commencing treatment the patient complained of sudden loss of vision in his left eye, which was his dominant eye because his right eye had age-related macular degeneration and correction of bilateral cataracts. $\mathrm{He}$ was referred to the Ocular Oncology Clinic at PMH. Anterior segments and intraocular pressure were found to be normal. Distant visual acuity was limited to hand waving. On examination, there was evidence of macular degeneration and area of choroidal infiltration consistent with metastatic disease extending from 2 to $7 \mathrm{o}^{\prime}$ clock in the right eye. The posterior segment of the left eye could not be visualized, but ultrasonography showed a lesion inferior to the optic nerve measuring $5 \mathrm{~mm}$ resulting in complete retinal detachment. These findings were consistent with bilateral metastases.

The patient was scheduled for urgent palliative radiotherapy to the bilateral orbits using parallel opposed fields 
$(5 \times 5 \mathrm{~cm})$ to a dose of $20 \mathrm{~Gy}$ in 5 fractions over 5 consecutive days (Fig. 1, top). Radiotherapy to the pelvis and bladder was continued; however, the case was now palliative with systemic metastases and the total dose was reduced to 50 Gy in 25 fractions over 5 weeks with chemotherapy discontinued at week 4 . The treatment was well tolerated by the patient.

At follow-up 54 months after treatment, the patient remained well with no symptoms or signs of local or distant recurrence. Follow-up MRI of the pelvis 54 months posttreatment demonstrated no evidence of tumour recurrence or metastatic disease. On review at the Ocular Oncology Clinic, the patient's visual acuity was stable at hand motions bilaterally. Bilateral fundus examination revealed no evidence of choroidal metastases. Ultrasonography of the left eye demonstrated a small area in the macular area measuring $1.5 \mathrm{~mm}$ in height with low internal reflectively and a hypoechoic area, which was unchanged from previous examinations and thought to probably represent scar tissue (Fig. 1, bottom). The patient continues in regular follow-up at $\mathrm{PMH}$.

\section{Discussion}

The first case of choroidal metastasis was reported by Perls in $1872 .{ }^{2}$ Choroidal metastases are the most common intraocular malignancy seen in adults, with breast and lung cancers accounting for most of these. Choroidal metastases secondary to genitourinary tract malignancies are less common with prostate and kidney cancer accounting for less than $5 \%$, respectively. ${ }^{1,3,4}$

Choroidal metastases secondary to urothelial cancers are extremely rare. Hart ${ }^{5}$ reported 2 cases of ocular metastases from carcinoma of the urinary bladder in 1962. There have been only a handful of cases of choroidal metastases from urothelial cancers reported in the literature since..$^{6-12}$ Choroidal metastases in this setting are typically seen in association with disseminated disease; however, there have been cases in which metastatic eye disease constituted the initial clinical manifestation of disease progression. ${ }^{8}$ Our case is unusual in that to date this has been the only site of metastatic disease.
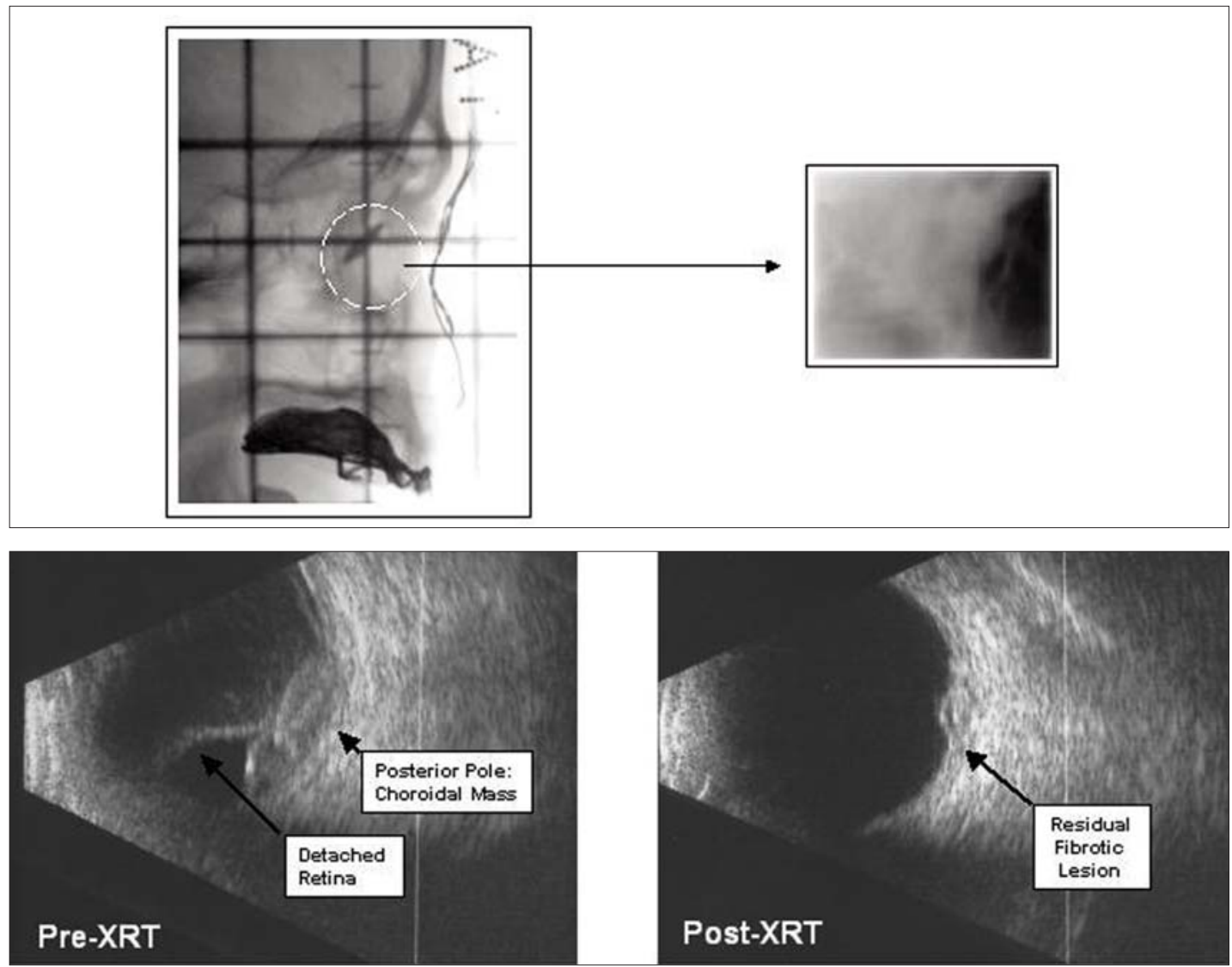

Fig. 1. Radiation simulation film showing small lateral rectangular field and orbit (dashed lines, left) and final radiotherapy treatment port film (right) to treat bilateral choroidal metastases with $20 \mathrm{~Gy}$ in 5 fractions (top). Preradiotherapy (left) and postradiotherapy (right) orbital ultrasound image of choroidal metastases from bladder cancer (bottom). 
Patients with choroidal metastases commonly present with decreased visual acuity. Diagnosis is based on clinical findings with metastases typically appearing as solid, flat, plaque-like, yellow-brown lesions. ${ }^{13}$ Associated retinal detachment is often seen. ${ }^{13,14} \mathrm{~A}$ thorough ophthalmological evaluation is required and additional investigations such as ultrasonography, computed tomography, MRI and fluorescein angiography can assist in the diagnosis. Tissue diagnosis (fine needle aspirate or wedge biopsy) should only be undertaken in difficult diagnostic situations in which this is likely to alter management, because of the high risk of complications and of seeding along the biopsy track. ${ }^{11,14}$ A detailed metastatic work up should be performed to assess the burden of disease and exclude other occult primaries.

The management of choroidal metastases is individualized to the patient with careful consideration to the performance status, burden of disease and life expectancy. The aim of management is to preserve or improve visual function, relieve discomfort and maintain quality of life. Treatment options include systemic chemotherapy in the presence of disseminated disease and/or local therapy. Local therapeutic modalities include EBRT, plaque brachytherapy, laser photocoagulation, surgical resection and cryosurgery. ${ }^{15}$

Radiotherapy is known to provide effective local palliation. Retrospective series of patients with choroidal metastases treated with EBRT report improvement or stablization of vision in $57 \%-62 \%$ of patients. ${ }^{14,16}$ In a series of 188 patients treated with EBRT reported by Rudoler and colleagues ${ }^{16} 36 \%$ of legally blind eyes regained useful vision and $93 \%$ experienced no clinical evidence of tumour progression with a globe preservation rate of $98 \%$. A prospective German Cancer Society study ${ }^{17}$ of 50 patients with choroidal metastases secondary to breast cancer treated with EBRT to 40 Gy in 20 fractions demonstrated stabilization or improvement in visual acuity in $86 \%$ of patients. A complete or partial response, as measured by ultrasonography, was seen in $63 \%$ of patients. Severe side effects (retinal detachment and optic neuropathy) occurred in 2 patients with a median follow-up of 5.8 months. In 1 patient this was possibly related to disease progression.

In previous case reports of patients with choroidal metastases secondary to urothelial cancers, a combination of systemic chemotherapy (i.e., methotrexate, vinblastine, adriamycin and cisplatin) and local radiotherapy to the orbits has been used. Despite aggressive treatment the natural history of choroidal metastases is one of rapid disease progression with patients often dying within 6 months of diagnosis. . $^{8,9,11}$

\section{Conclusion}

Although extremely rare, the possibility of choroidal metastases should be considered in a patient with a history of urothelial cancer presenting with the new onset of eye symptoms. The prognosis is usually extremely poor. Radiotherapy to the orbits provides good local palliation. Our patient is somewhat atypical in that at last follow-up he remained well without evidence of disease progression, local or distant, 4 years after completing treatment.

From the *Department of Radiation Oncology, Peter MacCallum Cancer Centre, Melbourne, Victoria, Australia and the ${ }^{\dagger}$ Department of Radiation Oncology, Princess Margaret Hospital (University Health Network), University of Toronto, Toronto, Ont.

This article has been peer reviewed.

Competing interests: None declared.

\section{References}

1. Shields CL, Shields JA, Gross NE, et al. Survey of 520 eyes with uveal metastases. Ophthalmology 1997;104:1265-76.

2. Perls M. Contributions to pathology of tumours. Virchows Arch Pathol Anat 1872;56:437.

3. Ferry AP, Font RL. Carcinoma metastatic to the eye and orbit. A clinicopathological study of 227 cases. Arch Ophthalmol 1974;92:276-86.

4. Freedman MI, Folk JC. Metastatic tumour to the eye and orbit: patient survival and clinical characteristics. Arch Ophthalmol 1987;105:1215-9.

5. Hart WM. Metastatic carcinoma in the eye and orbit. Int Ophthalmol Clin 1962;2:465-82.

6. Gordon HL, Munro R. Ocular metastasis of bladder cancer. South Med J 1974;67:745-6.

7. Resnick MI, $0^{\prime}$ Conor VJ Jr, Grayhack JT. Metastases to the eye from transitional cell carcinoma of the bladder. J Urol 1975; 114:722-4.

8. Cieplinski W, Ciesielski TE, Haine C, et al. Choroid metastases from transitional cell carcinoma of the bladder: a case report and a review of the literature. Cancer 1982;50:1596-600.

9. Atta HR. Presumed metastatic transitional cell carcinoma of the choroids. Br I Ophthalmol 1983; 67:830-3.

10. Pe'er J, Zimmerman LE. Ocular metastases from transitional cell carcinoma of the urinary tract. Graefes Arch Clin Exp Ophthalmol 1984;221:137-41.

11. Nabi G, Dadeya S, Dogra PN, et al. Eye metastasis form urothelial tumours. Int Urol Nephrol 2002; 34:51-4.

12. Chong JT, Mick A. Choroidal metastasis: case reports and review of the literature. Optometry 2005;76:293-301

13. Merrill CF, Kaufman DI, Dimitrov MV. Breast cancer metastatic to the eye is a common entity. Cancer 1991;68:623-7.

14. Rosset A, Zografos L, Coucke P, et al. Radiotherapy of choroidal metastases. Radiother Oncol 1998; $46: 263-8$

15. Amer R, Pe'er J, Chowers I, et al. Treatment options in the management of choroidal metastases. Ophthalmologica 2004;218:372-7.

16. Rudoler SB, Shields CL, Corn BW, et al. Functional vision is improved in the majority of patients treated with external-beam radiotherapy for choroid metastases: a multivariate analysis of 188 patients. J Clin Oncol 1997;15:1244-51.

17. Wiegel T, Bottke D, Kreusel KM, et al. External beam radiotherapy of choroidal metastases-final results of a prospective study of the German Cancer Society (ARO 95-08). Radiother Oncol 2002;64:13-8.

Correspondence: Dr. Robert G. Bristow, Radiation Medicine Program, Princess Margaret Hospital, 610 University Ave., Toronto ON M5G 2M9; fax 416 946-4586; rob.bristow@rmp.uhn.on.ca 